\title{
Maxillary Artery runs medial to the mandibular nerve unilaterally: Case report and Literature Review
}

\author{
Yonatan Schwartz ${ }^{* 1}$, Vindhya J. Rao ${ }^{* 1}$, Lesly Sanchez Alvarez ${ }^{{ }^{1}}$, Benjamin \\ Wajsberg ${ }^{* 1}$, Priti Mishall ${ }^{1}$, Sherry A. Downie ${ }^{1}$, Adi Pinkas $* 1$. \\ ${ }^{1}$ Department of Anatomy and Structural Biology, Albert Einstein College of Medicine, 1300 \\ Morris Park Avenue, F620S, Bronx, NY 10461
}

\section{ABSTRACT}

\begin{abstract}
Background: The maxillary artery supplies the deep structures of the face and cranium. The maxillary artery and the mandibular nerve form a clinically important relation in the infratemporal fossa. Variations of the maxillary artery may complicate dental, oral, and maxillofacial surgeries in this region.

Methods: During routine academic dissection of a 91-year-old Caucasian female cadaver, the pterygoid segment of the left maxillary artery was found medial to the posterior division of the mandibular nerve in the infratemporal fossa.

Results: The maxillary artery coursed superior to the point of division of the mandibular nerve and remained undivided.

Conclusion: This rare unilateral variation will be of interest to anatomists and clinicians, as an understanding of anatomical variations of the maxillary artery can help decrease the risk of hemorrhage and other surgical complications.

KEY WORDS: Maxillary artery, Mandibular nerve, Lateral pterygoid muscle, Infratemporal Fossa.
\end{abstract}

Corresponding Author: Adi Pinkas, Department of Anatomy and Structural Biology, Albert Einstein College of Medicine, 1300 Morris Park Avenue, F620S, Bronx, NY 10461

E-Mail: benjamin.wajsberg@einsteinmed.org; adi.pinkas@einsteinmed.org

\begin{tabular}{|c|c|c|c|}
\hline Access this Article online & \multicolumn{3}{|c|}{ Journal Information } \\
\hline \multirow[t]{3}{*}{ Quick Response code } & \multicolumn{3}{|c|}{$\begin{array}{l}\text { International Journal of Anatomy and Research } \\
\text { ISSN (E) 2321-4287 | ISSN (P) 2321-8967 } \\
\text { https://www.ijmhr.org/ijar.htm } \\
\text { DOI-Prefix: } \text { https://dx.doi.org/10.16965/ijar }\end{array}$} \\
\hline & \multicolumn{3}{|c|}{ Article Information } \\
\hline & $\begin{array}{l}\text { Received: } 02 \text { Apr } 2021 \\
\text { Peer Review: } 03 \text { Apr } 2021 \\
\text { Revised: None }\end{array}$ & $\begin{array}{l}\text { Accepted: } 10 \text { May } \\
\text { Published (O): } 05 \\
\text { Published (P): } 05\end{array}$ & $\begin{array}{l}21 \\
2021 \\
2021\end{array}$ \\
\hline
\end{tabular}

\section{INTRODUCTION}

The maxillary artery and its branches are the major arterial supply to the deep structures of the face and anterior cranium. The maxillary artery, which is the larger of the two terminal branches of the external carotid artery, originates posterior to the neck of the mandible, travels anteriorly and deep to the neck of the mandibular condyle to enter the infratemporal fossa [1].

Within the infratemporal fossa, the maxillary artery is described by its relation to the lateral pterygoid muscle as having three segments. The mandibular or first segment gives branches that supply the lower mandible and meninges among other areas. They are the deep auricular, anterior tympanic, middle meningeal, accessory meningeal, and inferior alveolar arteries $[1,2]$.

The pterygoid or second maxillary segment courses anteromedially across the lateral pterygoid muscle and gives the following branches: deep temporal, pterygoid, masseteric, and buccal arteries [2]. This segment can be superficial or deep to the lateral pterygoid, with the superficial course 
seen in $60 \%$ of cases [2]. It can also travel between the two heads of the lateral pterygoid [3]. The pterygopalatine or third segment runs anteromedial to the lateral pterygoid and gives rise to the posterior superior alveolar, infra-orbital, greater palatine, artery of pterygoid canal, and pharyngeal arteries, then terminates in the pterygopalatine fossa as the sphenopalatine artery. Knowledge of the maxillary artery and its branches is important in dental, oral, and maxillofacial surgeries [4]. Several cadaveric studies have documented variations in the maxillary artery and/or its branches [5-7]. The present case documents a rare variation of the left maxillary artery in which it coursed medial to the mandibular division of the trigeminal nerve and its branches.

\section{METHODS}

Bilateral infratemporal regions of a 91-yearold Caucasian female cadaver were dissected during a routine laboratory session in an academic setting. The zygomatic arch was detached from the skull and reflected inferiorly with the masseter muscle. The coronoid process of the mandible was transected, and the temporalis muscle was reflected superiorly. Finally, the infratemporal fossa was accessed by sawing through the mandibular ramus at the level of the mandibular foramen and removing the superior part of the ramus and the attached lateral pterygoid muscle. The infratemporal fossa was cleared of muscle and fascial remnants and arteries and nerves were identified.

\section{RESULTS}

Left Infratemporal Fossa: As expected, the left maxillary artery branched from the external carotid artery posterior to the mandibular ramus and travelled medially giving multiple branches. The inferior alveolar and middle meningeal branches were identified originating from the first (mandibular) segment of the maxillary artery (Figure 1). Careful blunt dissection revealed that the second (pterygoid) segment of the maxillary artery travelled medial to the mandibular nerve (Figure 1) before continuing anteromedially towards the pterygomaxillary fissure.

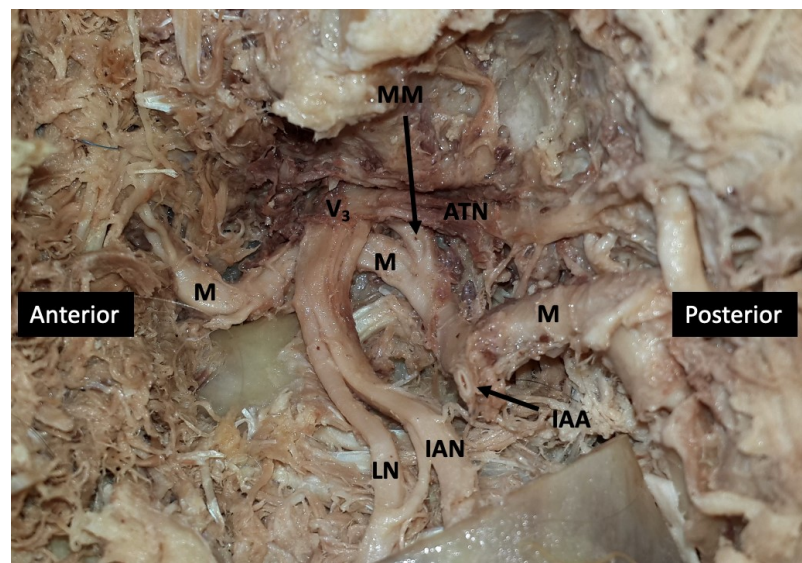

Fig. 1: Dissection of left infratemporal fossa. ATN auriculotemporal nerve, IAN inferior alveolar nerve, LN lingual nerve, $\mathrm{V}_{3}$ posterior division of mandibular nerve, $\mathrm{M}$ maxillary artery, MM middle meningeal artery, IAA inferior alveolar artery (cut).

Right Infratemporal Fossa: The right maxillary artery took a superficial course in relation to the lateral pterygoid muscle and therefore was lateral to the mandibular nerve and its branches. (Figure 2). As expected, the right inferior alveolar artery descended posterior to the right inferior alveolar nerve (Figure 2).

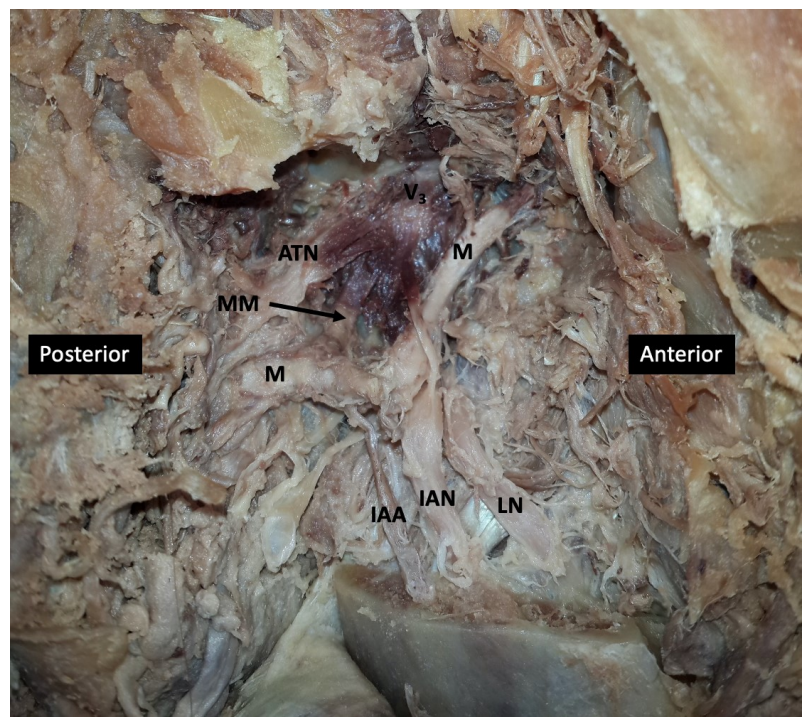

Fig. 2: Dissection of right infratemporal fossa. ATN auriculotemporal nerve, IAN inferior alveolar nerve, LN lingual nerve, $\mathrm{V}_{3}$ posterior division of mandibular nerve, $\mathrm{M}$ maxillary artery, MM middle meningeal artery, IAA inferior alveolar artery.

\section{DISCUSSION}

Several authors have described variations of the maxillary artery. Tadokoro et al. [9] described a divided maxillary artery in which one branch traveled medial to the lateral pterygoid muscle while the other traveled laterally. In addition, Claire et al. [6] reported a divided and reunited maxillary artery that formed a loop around the lateral pterygoid 
within the infratemporal fossa. Pretterkliber et al. [8] presented a maxillary artery that branched from the external carotid artery with the facial artery bilaterally. Furthermore, Maeda et al. [7] detailed a maxillary artery that ran directly through the lateral pterygoid muscle.

While there are varying presentations of the maxillary artery within the literature, our finding - a maxillary artery coursing medial to the mandibular nerve - appears to be a rare occurrence. In a study of 104 adult Japanese cadavers, Maeda et al. [7] reported a unilateral maxillary artery that coursed medial to the mandibular nerve. However, that maxillary artery was observed to pass more inferiorly in the infratemporal fossa placing it medial to the inferior alveolar nerve (IAN) and lingual nerve (LN) branches. In the present case, in contrast, the maxillary artery was located superior to the point of division of the mandibular nerve into the IAN and LN branches. Although in both cases the maxillary artery is medial to the mandibular nerve and its branches, it is important to recognize that the maxillary artery can appear in different locations in reference to the mandibular nerve. These differences can impact the pathway taken by the branches of the maxillary artery.

Additionally, Maeda et al. [7] referenced a case report by Tanaka et al. [9] that described a maxillary artery that curved superiorly in the infratemporal fossa and passed through a loop in the auriculotemporal nerve, then continued medial to the mandibular nerve. Unfortunately, we were unable to access this paper and our attempts to contact the authors have been unsuccessful.

It is important to note that Pretterklieber et al. [10] found a maxillary artery medial to the mandibular branches in $3.9 \%$ of 102 cadavers. However, this paper is rarely referenced in the literature. Perhaps this variation of the maxillary artery has garnered less interest from anatomists in recent years. Or perhaps this increased finding of an extremely rare case is due to a small and non-diverse sample size consisting of 101 individuals born in the eastern part of Austria and one individual born in

\section{Great Britain.}

Other variations of the maxillary artery in relation to the mandibular nerve have been reported. Tadokoro et al. [11] detailed a left maxillary artery divided into lateral and medial branches in relation to the lateral pterygoid muscle. The medial branch coursed medial to the posterior division of the mandibular nerve, similar to the present case. In addition, Pretterklieber et al. [8] reported finding the pterygoid segments of bilateral maxillary arteries deep to the lower head of the lateral pterygoid and the mandibular nerve. The right maxillary artery coursed deep to the inferior alveolar and buccal nerves but was superficial to the lingual nerve. The left maxillary artery was encircled by the left inferior and lingual nerves displaying two roots and ran deep to the buccal nerve. Furthermore, Verma et al. [4] reported a case of bilateral maxillary arteries medial to the posterior division of the mandibular nerve. Both maxillary arteries passed through loops created by two roots of the auriculotemporal nerve and the posterior division of the mandibular nerve before coursing medial to the posterior division of the mandibular nerve. Although it may seem unlikely for this anomaly to be reported bilaterally, symmetrical course of the right and left maxillary arteries is quite common, reported as $75 / 80(93.8 \%)$ by lkakura et al. [12] and 44/47 (93.6\%) by Takarada [13]. In contrast, the present case demonstrates a unilateral variation.

A comprehensive awareness of the numerous variations in the route of the maxillary artery and their relation to branches of the mandibular nerve is crucial when performing surgery in the infratemporal fossa. The maxillary artery, for example, makes an important contribution to the arterial supply of the nasal cavity, and its ligation is important for controlling epistaxis [14, 15]. Anatomical variations in the maxillary artery, like the case presented here, may complicate major procedures such as maxillary osteotomy, maxillectomy, tumor removal as well as simple procedures such as inferior alveolar nerve block for dental anesthesia [4, 6].

Awareness of these variations may help 
decrease the risk of hemorrhage and unnecessary complications $[4,8,16]$.

Additionally, an understanding of the anatomical variations of the maxillary artery is crucial for successful implementation of new procedures. The maxillary artery is gaining attention in neurosurgery, particularly in therapeutic techniques such as extracranialintracranial (EC-IC) bypass, intracranial endonasal approaches and endovascular procedures [5]. Due to close proximity with the cranial base, various studies are also investigating the ability of the maxillary artery to function as the arterial donor in the repair of internal carotid artery dissections and aneurysms [15].

Several reports of variations of the maxillary artery in the infratemporal fossa can be found in the existing literature. The present case, in which the second segment of the maxillary artery travelled medial to the mandibular nerve, is unique because it courses superior to the point of division of the mandibular nerve and it presents unilaterally.

\section{ACKNOWLEDGEMENTS}

The authors acknowledge and gratefully thank the individual whose body and tissues were used in this study for the advancement of physician education and patient care.

\section{Conflicts of Interests: None}

\section{REFERENCES}

[1]. Moore, K.L.A., A. M. R.; Dalley II, A.F. , Essential clinical anatomy 2015, Philadelphia: Lippincott Williams \& Wilkins.

[2]. Tanoue, S., et al., Maxillary artery: functional and imaging anatomy for safe and effective transcatheter treatment. Radiographics, 2013;33(7):e209-24.

[3]. Dennison, J., A. Batra, and P. Herbison, The maxillary artery and the lateral pterygoid muscle: the New Zealand story. Oral Surg Oral Med Oral Pathol Oral Radiol Endod, 2009;108(5):26-9.

[4]. Verma, S., et al., Unique variation in the course of maxillary artery in infratemporal fossa: a case report. Surg Radiol Anat, 2014;36(5):507-9.
[5]. Alvernia, J.E., et al., The maxillary artery and its variants: an anatomical study with neurosurgical applications. Acta Neurochir (Wien), 2017;159(4):655-664.

[6]. Claire, P.G., et al., Divided and reunited maxillary artery: developmental and clinical considerations. Anat Sci Int, 2011;86(4):232-6.

[7]. Maeda, S., et al., Variations in the course of the maxillary artery in Japanese adults. Anat Sci Int, 2012;87(4):187-94.

[8]. Pretterklieber, M.L., E.B. Krammer, and R. Mayr, A bilateral maxillofacial trunk in man: an extraordinary anomaly of the carotid system of arteries. Acta Anat (Basel), 1991; 141(3):206-11.

[9]. Tanaka S, I.K., Tanaka R, Kawai K, Shimoda S, Kodera $H$, Goto M, Kawasaki K, Sato T, Maxillary artery passing among the branches from the mandibular nerve in a Japanese man. Tsurumi Univ Dent J, 2003;29:187-191.

[10]. Pretterklieber, M.L., C. Skopakoff, and R. Mayr, The human maxillary artery reinvestigated: I. Topographical relations in the infratemporal fossa. Acta Anat (Basel), 1991;142(4):281-7.

[11]. Tadokoro, O., et al., A case of a divided maxillary artery in the infratemporal fossa. Okajimas Folia Anat Jpn, 2008;85(3):97-101.

[12]. Ikakura, K., On the origin, course and distribution of the maxillary artery in Japanese. Arch Dept of Anat Tokyo Dent Coll. , 1961;18:91-122.

[13]. Takarada, T., Anatomical studies on the maxillary artery. Shikwa gakuho 1958;58:1-20.

[14]. Polev, G.A., et al., Intraoral Endoscopic Ligation of Maxillary Artery in the Infratemporal Fossa. J Craniofac Surg, 2019;30(1):137-140.

[15]. Uysal II, B.M., Dogan NU, Seker M, Ziylan T Clinical significance of maxillary artery and its branches: a cadaver study and review of literature. Int J Morphol, 2011;29(4):1274-1281.

[16]. Toure, G., Distribution of the maxillary artery in the deep regions of the face and the maxilla: Clinical applications. J Plast Reconstr Aesthet Surg, 2019;72(6):1020-1024.

\section{How to cite this article:}

Yonatan Schwartz, Vindhya. Rao, Lesly Sanchez Alvarez, Benjamin Wajsberg, Priti Mishall, Sherry A. Downie, Adi Pinkas. Maxillary Artery runs medial to the mandibular nerve unilaterally: Case report and Literature Review. Int J Anat Res 2021;9(2.3):8008-8011. DOI: 10.16965/ijar.2021.127 